\title{
Validation of MRC Centre MRI calf muscle fat fraction protocol as an outcome measure in CMT1A
}

Jasper M. Morrow, PhD, Matthew R.B. Evans, MBBS, Tiffany Grider, MS, Christopher D.J. Sinclair, PhD, Daniel Thedens, PhD, Sachit Shah, MD, Tarek A. Yousry, Dr Med Habil, Michael G. Hanna, MD,

Peggy Nopoulos, MD, John S. Thornton, PhD, Michael E. Shy, MD, and Mary M. Reilly, MD

Neurology ${ }^{\circledR}$ 2018;91:e1125-e1129. doi:10.1212/WNL.0000000000006214

\section{Abstract}

\section{Objective}

To translate the quantitative MRC Centre MRI protocol in Charcot-Marie-Tooth disease type 1A (CMT1A) to a second site; validate its responsiveness in an independent cohort; and test the benefit of participant stratification to increase outcome measure responsiveness.

\section{Methods}

Three healthy volunteers were scanned for intersite standardization. For the longitudinal patient study, 11 patients with CMT1A were recruited with 10 patients rescanned at a 12 month interval. Three-point Dixon MRI of leg muscles was performed to generate fat fraction (FF) maps, transferred to a central site for quality control and analysis. Clinical data collected included CMT Neuropathy Score.

\section{Results}

Test-retest reliability of FF within individual healthy calf muscles at the remote site was excellent: intraclass correlation coefficient 0.79 , limits of agreement -0.67 to $+0.85 \% \mathrm{FF}$. In patients, mean calf muscle FF was $21.0 \%$ and correlated strongly with disease severity and age. Calf muscle FF significantly increased over 12 months $(+1.8 \pm 1.7 \% \mathrm{FF}, p=0.009)$. Patients with baseline $\mathrm{FF}>10 \%$ showed a 12 -month FF increase of $2.9 \% \pm 1.3 \%$ (standardized response mean $=2.19$ ).

\section{Conclusions}

We have validated calf muscle FF as an outcome measure in an independent cohort of patients with CMT1A. Responsiveness is significantly improved by enrolling a stratified patient cohort with baseline calf FF $>10 \%$.

\author{
Correspondence \\ Prof. Reilly \\ m.reilly@ucl.ac.uk
}




\section{Glossary}

CMT = Charcot-Marie-Tooth disease; CMT1A = Charcot-Marie-Tooth disease type 1A; CMTES = Charcot-Marie-Tooth examination score; CMTNS = Charcot-Marie-Tooth Neuropathy Score; FF = fat fraction; SRM = standardized response mean.

Charcot-Marie-Tooth disease (CMT) or hereditary motor and sensory neuropathy is a common disease with a prevalence of 1 in 2,500. CMT causes progressive distal weakness, sensory loss, and disability, with CMT type 1A (CMT1A) accounting for more than half of all cases. ${ }^{1}$ There is currently no disease-modifying therapy although a number of promising agents are in development. Trials of vitamin $\mathrm{C}$ were negative ${ }^{2,3}$ but highlighted the need for responsive outcome measures.

Quantitative skeletal muscle MRI has been widely studied as an outcome measure in muscle diseases, ${ }^{4,5}$ and we recently reported high responsiveness over 12 months in CMT1A in a UK cohort. ${ }^{6}$ Intramuscular fat accumulation occurs as a common feature of chronic pathology in muscle whether the primary pathogenesis is neurogenic or myopathic, and can be quantified with MRI using fat-water separation methods such as the 3-point Dixon technique. ${ }^{5}$ In our previous study, this provided the most responsive outcome measure with a 12month total calf muscle fat fraction (FF) increase of $1.2 \% \pm$ $1.5 \%(p=0.008)$ and standardized response mean (SRM) of 0.83 , far exceeding that of any clinical outcome measure. ${ }^{6}$ However, it was recognized that this required further validation in an independent CMT1A cohort before application in clinical trials.

We aimed to translate our MRC Centre MRI protocol to a second site for validation in an independent patient cohort. We also tested the potential of stratification based on baseline muscle FF to increase outcome measure responsiveness.

\section{Methods}

MRI was performed at the University of Iowa, Iowa City, with anonymized MRI data transferred for analysis at the MRC Centre for Neuromuscular Diseases, UCL Institute of Neurology, London, UK.

Eleven patients with genetically confirmed CMT1A were recruited for examination at baseline and 12 months. Age, disease duration, and the CMT Neuropathy Score (CMTNS) (a composite disease severity scale ranging from $0=$ normal to 36 = maximum severity) were recorded. For longitudinal comparisons, the examination score (CMTES) component was used. The Rasch-modified CMTNS and CMTES were calculated. ${ }^{7}$

The MRC Centre MRI protocol, identical to that used previously, ${ }^{4}$ was performed using a similar scanner (Siemens
3T TIM TRIO; Siemens Medical Solutions, Malvern, PA). In brief, calf-level 3-point Dixon MRI was performed centered $130 \mathrm{~mm}$ distal from the knee joint. ${ }^{8}$ Muscle-group cross-sectional regions of interest (figure 1) were drawn by a single observer (J.M.M.) on the right calf muscle on the central slice of the 3.45-millisecond Dixon acquisition to allow extraction of the corresponding combined all-musclegroup mean FF.

For remote-site quality-control qualification, 3 healthy volunteers were scanned with 2 undergoing repeat scanning on a different day to assess test-retest reliability, referenced to similar measurements performed in London. 9,10

Statistical analysis was performed using SPSS Statistics 20.0 (IBM Corp., Armonk, NY). Group data were compared using

Figure 1 Quantitative MRI in a range of disease severity in Charcot-Marie-Tooth disease type 1A

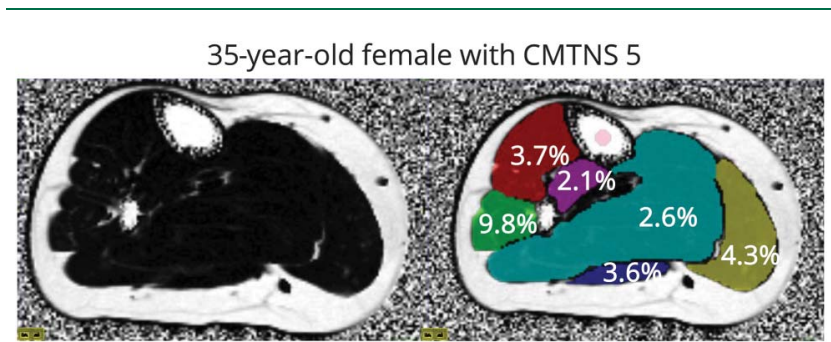

43-year-old male with CMTNS 19

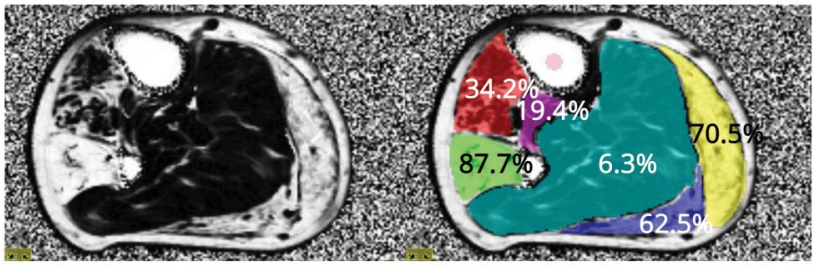

64-year-old male with CMTNS 23

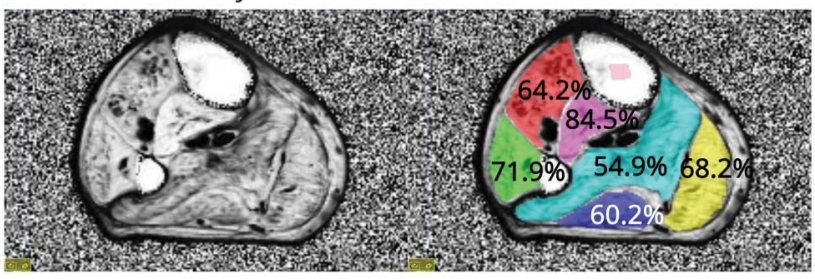

Axial fat fraction map of the right calf $130 \mathrm{~mm}$ distal to the lateral tibial plateau is shown on the left. Regions of interest (red: tibialis anterior/extensor hallucis longus; green: peroneus longus; purple: tibialis posterior; light blue: soleus; dark blue: lateral gastrocnemius; yellow: medial gastrocnemius) and respective mean fat fraction values are overlaid on the right. In the top patient, there is a slight increase in fat fraction in peroneus longus only. In the middle row, marked involvement is seen with endstage involvement of peroneus longus and both heads of the gastrocnemius. In the bottom row, there is severe involvement of all muscle groups. CMTNS = Charcot-Marie-Tooth Neuropathy Score. 
student 2-tailed $t$ tests, longitudinal change was assessed using paired $t$ test, and MRI-clinical associations were assessed using Spearman rank correlation. Outcome measure responsiveness was assessed using the SRM (mean change divided by the change SD).

To investigate stratification, the remote-site data were combined with that from our previous study ${ }^{4}$ in a clinically similar CMT1A patient group (20 patients with CMT1A, 11 men, mean age 42.8 years, mean CMTES 8.0). Twelve-month FF changes and SRMs were calculated for patients with baseline overall muscle FFs above and below a threshold of $10 \%$, for both sites separately and combined.

\section{Data availability}

Anonymized data will be shared by request from any qualified investigator.

\section{Standard protocol approvals, registrations, and patient consents}

Both sites' ethical review boards approved the study and all participants gave written informed consent.

\section{Results}

Of the 11 patients with CMT1A assessed at baseline (6 women, 5 men, mean age 41, range 24-64 years; mean baseline CMTNS 15.2, SD 5.9, range 5-23), 10 underwent repeat assessment at median interval 1.03 years.

The remote-site qualification FF measurements showed excellent test-retest reliability (intraclass correlation coefficient 0.79; Bland-Altman limits of agreement -0.67 to $+0.85 \% \mathrm{FF})$. No images were affected by artifact, and repeat-scanning block positions were sufficiently consistent that the same numerical slice could be used for follow-up analysis in all cases.

Figure 1 shows FF in participants with mild, moderate, and severe CMTNS. The patients' baseline mean overall calf muscle $\mathrm{FF}$ was $21 \%$ (median 8.7, interquartile range 5.2-34.1). Peroneus longus had the highest mean FF (37.9\%), with tibialis posterior the lowest $(15.0 \%)$. Overall calf muscle FF correlated strongly with age $(\rho=0.71, p=0.01)$, CMTNS $(\rho=0.695, p=0.03)$, and Rasch-modified CMTNS $(\rho=0.81$, $p=0.004)$.

Overall calf muscle FF increased over 12 months (+1.8 \pm 1.7 $\% \mathrm{FF}, p=0.009$ ), with increases in peroneus longus and lateral gastrocnemius also significant (table). Calf muscle FF showed high responsiveness (SRM 1.04), which improved on stratification: patients with baseline FF $>10 \%$ showed a 12 -month increase of $2.9 \pm 1.3 \% \mathrm{FF}(\mathrm{SRM}=2.19)$ (table, figure 2$)$. Compared with the subgroup with $<10 \%$ calf muscle FF, those with FF $>10 \%$ were older and more severely affected (mean age 50 years vs 34 years; mean Rasch-modified CMTES 18 vs 7). The Rasch-modified CMTES increased
Table Longitudinal change in calf-level muscle FF by muscle and stratified by baseline FF

\begin{tabular}{lllll}
\hline & $\begin{array}{l}\text { Mean FF } \\
\text { change } \\
\text { over 1 } \mathbf{y}, \%\end{array}$ & SD, $\%$ & $\boldsymbol{p}$ & SRM \\
\hline $\begin{array}{l}\text { US cohort }(\mathbf{n}=\mathbf{1 0}) \text { by } \\
\text { muscle }\end{array}$ & 0.8 & 1.9 & 0.19 & 0.45 \\
\hline Tibialis anterior & 1.6 & 2.1 & 0.04 & 0.74 \\
\hline Peroneus longus & 3.7 & 3.1 & 0.005 & 1.19 \\
\hline Lateral gastrocnemius & 3.0 & 4.8 & 0.08 & 0.63 \\
\hline Medial gastrocnemius & 3.7 & 2.6 & 0.07 & 0.65 \\
\hline Soleus & 1.7 & 2.4 & 0.25 & 0.39 \\
\hline Tibialis posterior & 0.9 & 1.7 & 0.009 & 1.04 \\
\hline Overall & 1.8 & & & \\
\hline
\end{tabular}

Restricted to patients

with baseline FF $<10 \%$

\begin{tabular}{lcccc}
\hline UK cohort $(\mathbf{n = 1 2 )}$ & 0.6 & 1.2 & 0.09 & 0.53 \\
\hline US cohort $(\mathbf{n = 5 )}$ & 0.6 & 1.3 & 0.32 & 0.51 \\
\hline $\begin{array}{c}\text { UK + US combined } \\
(\mathbf{n}=\mathbf{1 7})\end{array}$ & 0.6 & 1.2 & 0.04 & 0.54 \\
\hline
\end{tabular}

Restricted to patients

with baseline FF $>10 \%$

\begin{tabular}{ccccc}
\hline UK cohort $(\mathbf{n}=\mathbf{5})$ & 2.7 & 1.1 & 0.005 & 2.49 \\
\hline US cohort $(\mathbf{n}=\mathbf{5})$ & 2.9 & 1.3 & 0.008 & 2.19 \\
\hline $\begin{array}{c}\text { UK + US combined } \\
(\mathbf{n}=\mathbf{1 0})\end{array}$ & 2.8 & 1.1 & $<0.001$ & 2.44 \\
\hline
\end{tabular}

Abbreviations: $F F=$ fat fraction; SRM = standardized response mean. $p$ : Significance 2 -tailed $t$ test.

over 12 months but this was not statistically significant $(+1.4$ $\pm 2.6, p=0.13$ ).

\section{Discussion}

In this study, we successfully translated our calf-muscle MRC Centre MRI protocol to an international remote site, and further validated the value of calf-level muscle fat-fraction measurement in an independent cohort of CMT1A patients with high reliability, strong baseline cross-sectional clinical correlations, and high responsiveness. Stratification of the cohort based on baseline FF further improved the SRM to 2.44 in the combined cohort, resulting in an estimated sample size by the Lehr formula ${ }^{8}$ of 11 in each study arm of a randomized controlled trial with 0.8 power to detect a $50 \%$ slowing of disease progression at $p<0.05$.

These data from an American cohort validate the results obtained in our original British CMT1A cohort ${ }^{4}$ (figure 2) and demonstrate international multisite trial readiness of quantitative lower limb MRI in patients with CMT1A. 


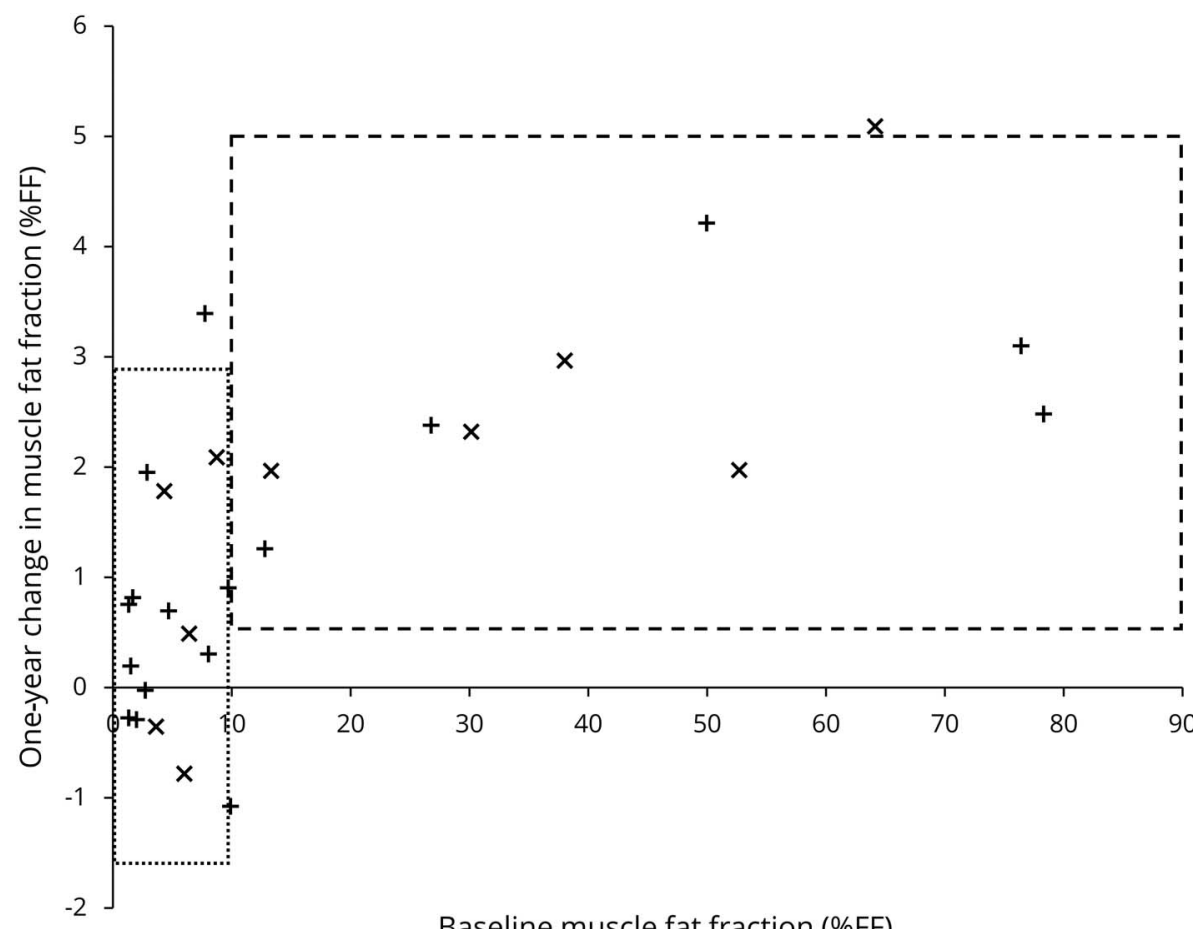

Baseline muscle fat fraction (\%FF) x: US patients (this study); +: UK patients. ${ }^{6}$ Boxes are $95 \%$ confidence intervals for the pooled data grouped by baseline calf muscle fat fraction greater than or less than $10 \%$. Fat accumulation is greater when baseline fat fraction is higher than $10 \%$, which is consistent across the 2 studies (table).
Utilization of a positioning protocol based on a fixed distance from the knee joint ${ }^{6}$ resulted in improved block positioning (10/10 scans within $1 \mathrm{~cm}$ of baseline in z-axis US vs $17 / 57$ UK), confirming the superiority of this method. The 3-point Dixon method is robust after a brief study setup phase with no regions of interest excluded from analysis because of artifact in this study. The study setup phase was possible without an inperson site visit with images transferred internationally for quality-control purposes.

Outcome measure criterion validity was demonstrated with both cohorts showing similar FF correlations with disease severity ( $\rho=0.81, p=0.004$ US with Rash-CMTS; $\rho=0.63, p$ $=0.003$ UK with CMTES $)$ and age $(\rho=0.84, p<0.001 \mathrm{UK} ; \rho$ $=0.71, p=0.01 \mathrm{US}$ ). Of note, the primary calf muscle FF outcome measure proposed previously ${ }^{6}$ showed similar increases $(+1.2 \pm 1.5 \% \mathrm{FF} \mathrm{UK},+1.8 \pm 1.7 \% \mathrm{FF}$ US $)$.

The SRM can be improved by participant stratification (figure 2 ): including only patients with baseline calf muscle FF $>10 \%$ yielded an SRM >2 consistently in the United States, United Kingdom, and combined cohorts (table), markedly higher than the nonstratified groups. The inverse square relationship of SRM to sample size implies a potential dramatic reduction resulting in patient numbers needed in an equivalently powered study from 83 to 11 in each arm. Researchers would need to consider a potential ceiling effect in the trial design as the calf muscle FF approached the maximum level with $78 \%$ the highest value obtained across the 2 studies. There was less responsiveness in those with low baseline FF at calf level. Further studies to examine responsiveness of FF assessed in the distal calf or foot musculature are in process to identify a more responsive biomarker in this younger, more mildly affected subgroup. However, MRI calf muscle FF provides a highly responsive outcome measure in an unselected adult CMT population.

We have confirmed the reliability, validity, and responsiveness of the MRC Centre MRI quantified calf muscle FF protocol as an outcome measure in an independent cohort of patients with CMT1A. Selection of study participants with increased baseline calf muscle FF provides a highly responsive biomarker in this patient group, suitable for utilization in multicenter international clinical trials.

\section{Author contributions}

Jasper M. Morrow: drafting/revising the manuscript, study concept or design, analysis or interpretation of data, accepts responsibility for conduct of research and will give final approval, statistical analysis. Matthew R.B. Evans: drafting/ revising the manuscript, accepts responsibility for conduct of research and will give final approval, acquisition of data. Tiffany Grider: drafting/revising the manuscript, accepts responsibility for conduct of research and will give final approval, study supervision. Christopher D.J. Sinclair: drafting/revising the manuscript, study concept or design, analysis or interpretation of data, accepts responsibility for conduct of research and will give final approval, acquisition of 
data. Daniel Thedens: drafting/revising the manuscript, analysis or interpretation of data, accepts responsibility for conduct of research and will give final approval, acquisition of data, study supervision. Sachit Shah: analysis or interpretation of data, accepts responsibility for conduct of research and will give final approval. Tarek A. Yousry: drafting/revising the manuscript, study concept or design, analysis or interpretation of data, accepts responsibility for conduct of research and will give final approval, acquisition of data, study supervision, obtaining funding. Michael G. Hanna: drafting/ revising the manuscript, study concept or design, accepts responsibility for conduct of research and will give final approval, study supervision, obtaining funding. Peg Nopoulos: drafting/revising the manuscript, accepts responsibility for conduct of research and will give final approval. John S. Thornton: drafting/revising the manuscript, study concept or design, accepts responsibility for conduct of research and will give final approval, acquisition of data, study supervision. Michael E. Shy: drafting/revising the manuscript, study concept or design, analysis or interpretation of data, accepts responsibility for conduct of research and will give final approval, acquisition of data, obtaining funding. Mary $\mathrm{M}$. Reilly: drafting/revising the manuscript, study concept or design, analysis or interpretation of data, accepts responsibility for conduct of research and will give final approval, acquisition of data, statistical analysis, study supervision, obtaining funding.

\section{Study funding}

This work was supported by the National Institute of Neurological Disorders and Stroke and Office of Rare Diseases (U54NS065712) and the MRC (grants G0601943 2008-2013 and MR/K000608/1 2013-2018). The authors gratefully acknowledge the capital and research support of the NIHR University College London Hospitals Biomedical Research Centre (2008-2013 and 2013-2018).

\section{Disclosure}

J. Morrow received funding from the Medical Research Council (MRC) and National Institute for Health Research (NIHR) University College London Hospitals Biomedical Research Centre during the conduct of this study. M. Evans received funding from the Medical Research Council during the conduct of this study. T. Grider reports no disclosures relevant to the manuscript. C. Sinclair received funding from the Medical Research Council during the conduct of this study. D. Thedens and S. Shah report no disclosures relevant to the manuscript. T. Yousry reports grants from MRC and NIHR University College London Hospitals Biomedical Research Centre during the conduct of the study and grants from Biogen Idec, GlaxoSmithKline, and Novartis outside the submitted work. M. Hanna reports grants from MRC and NIHR University College London Hospitals Biomedical Research Centre during the conduct of the study. P. Nopoulos reports no disclosures relevant to the manuscript. J. Thornton reports grants from MRC and NIHR University College London Hospitals Biomedical Research Centre during the conduct of the study and grants from Siemens Healthcare and GlaxoSmithKline and Engineering and Physical Sciences Research Council outside the submitted work. M. Shy received support from the Inherited Neuropathy Consortium (U54 NS065712), a collaboration between NCATS and NINDS. Dr. Shy also received funding from the Muscular Dystrophy Association and the Charcot-Marie-Tooth Association. M. Reilly received research support from the MRC, the National Institute of Neurological Disorders and Stroke, Office of Rare Diseases (U54NS065712), the National Institute for Health Research University College London Hospitals Biomedical Research Centre and CMT UK. Go to Neurology.org/ $\mathrm{N}$ for full disclosures.

Received February 15, 2018. Accepted in final form June 26, 2018.

\section{References}

1. Reilly MM, Murphy SM, Laurá M. Charcot-Marie-Tooth disease. J Peripher Nerv Syst2011;16:1-14.

2. Pareyson D, Reilly MM, Schenone A, et al. Ascorbic acid in Charcot-Marie-Tooth disease type 1A (CMT-TRIAAL and CMT-TRAUK): a double-blind randomised trial. Lancet Neurol 2011;10:320-328.

3. Lewis RA, McDermott MP, Herrmann DN, et al. High-dosage ascorbic acid treatment in Charcot-Marie-Tooth disease type 1A: results of a randomized, double-masked, controlled trial. JAMA Neurol 2013;70:981-987.

4. Janssen B, Voet N, Geurts A, van Engelen B, Heerschap A. Quantitative MRI reveals decelerated fatty infiltration in muscles of active FSHD patients. Neurology 2016;86: 1700-1707.

5. Burakiewicz J, Sinclair CDJ, Fischer D, Walter GA, Kan HE, Hollingsworth KG. Quantifying fat replacement of muscle by quantitative MRI in muscular dystrophy. J Neurol 2017;264:2053-2067.

6. Morrow JM, Sinclair CDJ, Fischmann A, et al. MRI biomarker assessment of neuromuscular disease progression: a prospective observational cohort study. Lancet Neurol 2016;15:65-77.

7. Sadjadi R, Reilly MM, Shy ME, et al. Psychometrics evaluation of Charcot-MarieTooth Neuropathy Score (CMTNSv2) second version, using Rasch analysis. J Peripher Nerv Syst 2014;19:192-196.

8. Fischmann A, Morrow JM, Sinclair CDJ, et al. Improved anatomical reproducibility in quantitative lower-limb muscle MRI. J Magn Reson Imaging 2014;4:1033-1038.

9. Morrow JM, Sinclair CDJ, Fischmann A, et al. Reproducibility, and age, body-weight and gender dependency of candidate skeletal muscle MRI outcome measures in healthy volunteers. Eur Radiol 2014;24:1610-1620.

10. Lehr R. Sixteen S-squared over D-squared: a relation for crude sample size estimates. Stat Med 1992;11:1099-1102. 


\section{Neurology}

\section{Validation of MRC Centre MRI calf muscle fat fraction protocol as an outcome measure in CMT1A}

Jasper M. Morrow, Matthew R.B. Evans, Tiffany Grider, et al.

Neurology 2018;91;e1125-e1129 Published Online before print August 17, 2018

DOI 10.1212/WNL.0000000000006214

This information is current as of August 17, 2018

\section{Updated Information \&} Services

References

Citations

Subspecialty Collections

\section{Permissions \& Licensing}

Reprints including high resolution figures, can be found at: http://n.neurology.org/content/91/12/e1125.full

This article cites 10 articles, 1 of which you can access for free at: http://n.neurology.org/content/91/12/e1125.full\#ref-list-1

This article has been cited by 3 HighWire-hosted articles: http://n.neurology.org/content/91/12/e1125.full\#\#otherarticles

This article, along with others on similar topics, appears in the following collection(s):

\section{All Genetics}

http://n.neurology.org/cgi/collection/all_genetics

MRI

http://n.neurology.org/cgi/collection/mri

Natural history studies (prognosis)

http://n.neurology.org/cgi/collection/natural_history_studies_prognosis

Peripheral neuropathy

http://n.neurology.org/cgi/collection/peripheral_neuropathy

Information about reproducing this article in parts (figures,tables) or in its entirety can be found online at:

http://www.neurology.org/about/about_the_journal\#permissions

Information about ordering reprints can be found online:

http://n.neurology.org/subscribers/advertise

Neurology ${ }^{\circledR}$ is the official journal of the American Academy of Neurology. Published continuously since 1951, it is now a weekly with 48 issues per year. Copyright Copyright ( 2018 The Author(s). Published by Wolters Kluwer Health, Inc. on behalf of the American Academy of Neurology.. All rights reserved. Print ISSN: 0028-3878. Online ISSN: 1526-632X.

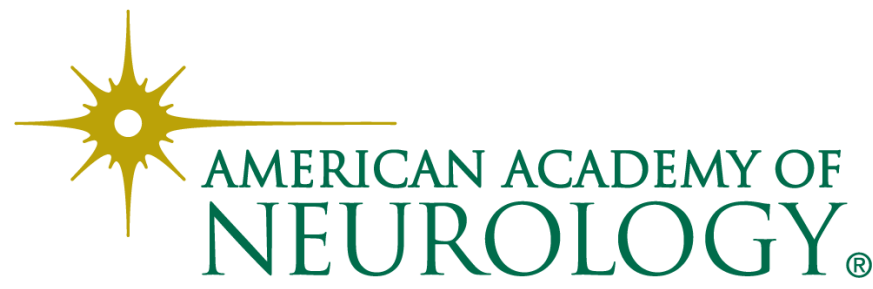

